\title{
KIC 4544587: An Eccentric, Short Period Binary with $\delta$ Scuti Pulsations and Tidally Excited Modes
}

\author{
Kelly Hambleton ${ }^{1}$, Don Kurtz ${ }^{1}$, Andrej Prša ${ }^{2}$, Steven Bloemen ${ }^{3}$, \\ and John Southworth ${ }^{4}$ \\ ${ }^{1}$ Jeremiah Horrocks Institute, University of Central Lancashire, Preston, PR1 2HE \\ email: kmh@uclan.ac.uk \\ ${ }^{2}$ Villanova University, Department of Astronomy and Astrophysics, 800 East Lancaster \\ Avenue, Villanova, PA $19085{ }^{3}$ Instituut voor Sterrenkunde, Katholieke Universiteit Leuven, \\ Celestijnenlaan 200D, B-3001 Leuven, Belgium ${ }^{4}$ Astophysics Group, Keele University, \\ Newcastle-under-Lyme, ST5 5BG, UK
}

\begin{abstract}
KIC 4544587 is an eclipsing binary star with clear signs of apsidal motion and indications of tidal resonance. The primary component is an early A-type $\delta$ Scuti variable, with a temperature of $8270 \pm 250 \mathrm{~K}$, whilst the secondary component is an early G-type main sequence star with a temperature of $6500 \pm 310 \mathrm{~K}$. The orbital period of this system is $2.18911(1) \mathrm{d}$, with the light curve demonstrating a hump after secondary minimum due to distortion and reflection. The frequency spectrum of the residual data (the original data with the binary characteristics removed) contains both pressure (p) and gravity (g) modes. Eight of the g modes are precise multiples of the orbital frequency, to an accuracy greater than $3 \sigma$. This is a signature of resonant excitation.
\end{abstract}

\section{Introduction}

In a binary system, where the components are in relatively close proximity to one another, the gravitational forces between the two components can induce tidal interactions. Moreover, if the binary is in an eccentric orbit, the tidal distortions become misaligned with respect to their instantaneous equipotential shapes (Hut 1980). This generates a torque between the two components which causes an exchange of orbital and rotational angular momentum, and thus causes the stellar rotational energy to dissipate.

In an eccentric binary system, the gravitational interactions between the two components vary as a function of phase. In some cases such interactions can cause one or both stellar components to oscillate as a result of the natural free eigenfrequencies of the star resonating with the dynamic tides (Aerts \& Hamanec 2004). The signature of these interactions are frequencies that are multiples (harmonics) of the orbital frequency.

\section{Overview}

KIC 4544587 is an eccentric, $e=0.28375(5)$, short-period binary system. The primary component is an early A star that is within the $\delta$ Scuti instability strip and the secondary component is an early $\mathrm{G}$ star. This object was selected as a likely candidate for tidally enhanced pulsations due to its $\delta$ Scuti component and the close proximity of the stars $\left(\sim 8 \mathrm{R}_{\odot}\right)$ at periastron. The system also has interesting orbital characteristics which include periastron brightening in the Kepler photometric light curve after secondary minimum; a feature that is indicative of an eccentric binary with its components in close proximity to each other (Maceroni et al. 2009). 


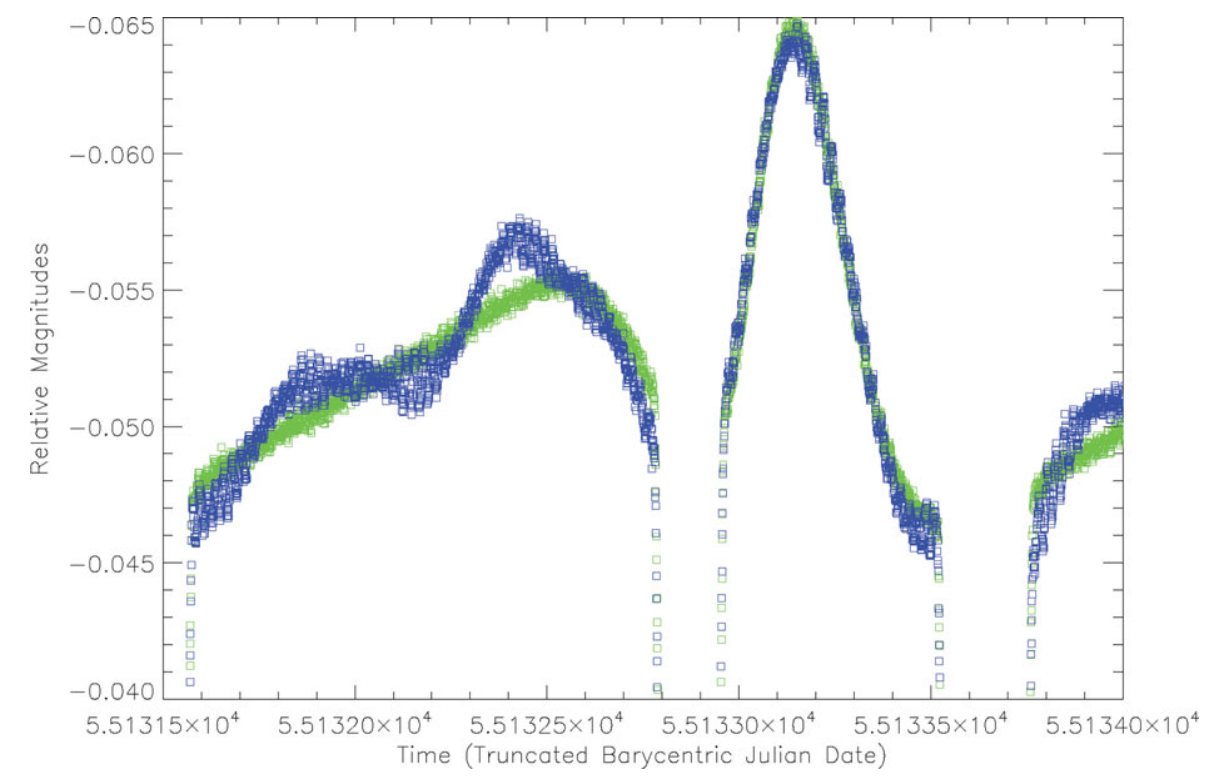

Figure 1. A comparison of the light curve with all the pulsation frequencies removed (green) and with all the frequencies except the harmonics of the orbital period removed (blue).

The Kepler photometric light curve that we studied consists of 46115 data points of both long $(29.4 \mathrm{~min})$ and short $(58.8 \mathrm{~s})$ cadence data. The orbital period of the binary system was found using the Phase Dispersion Minimization technique (Stellingwerf 1978) on the Quarter 3.2 data and was determined to be 2.1891142(5) d.

Subsequently, the modelling was carried out on the short cadence, phase binned data of Quarter 3.2 (which spans 30.04 d) using the Wilson-Devinney code (Wilson \& Devinney 1971). Frequency analysis was performed on the residuals (observed minus computed) by applying a Fourier transform combined with a least squares fit to the data. The identified frequencies were then pre-whitened from the original light curve, which was then remodelled. This enabled the determination of the binary characteristics without interference from the pulsations. This process was then repeated for three iterations.

Forty nine frequencies were identified in the residual data excluding the data taken during eclipse phases. These frequencies consist of 13 modes within the g mode regime $\left(\nu<5 \mathrm{~d}^{-1}\right)$ and 37 within the $\mathrm{p}$ mode regime $\left(30 \mathrm{~d}^{-1}<\nu<50 \mathrm{~d}^{-1}\right)$. Eight of the $\mathrm{g}$ mode frequencies were found to be multiples of the orbital frequency to an accuracy of 3 sigma or greater. In Fig. 1 the blue (darker) light curve depicts the original light curve with all the frequencies except the orbital harmonics removed and the green (lighter) light curve depicts the original light curve with all the identified frequencies removed. A plausible explanation for the remnant periodicity in the light curve is the resonant excitation of $g$ modes due to tidal interactions.

\section{References}

Abt, H. A. 2009, ApJ, 13828

Aerts, C. \& Harmanec, P. 2004, arXiv:astro-ph/0510344, 318325

Hut, P. 1980, A\&̈A, 92167

Maceroni, C., Montalbán, J., Michel, E., \& Harmanec, P. 2009, \& Prša, A. A\&̛A, 5081375

Wilson, R. E. \& Devinney, E. J. 1971, ApJ, 166605 